\title{
Geometry contrast enhancement for 3D point models using histogram modification
}

\author{
Jin-Woo Nam and Jae-Young Sim ${ }^{\text {a) }}$ \\ School of Electrical and Computer Engineering, UNIST \\ UNIST-gil 50, Eonyang-eup, Ulju-gun, Ulsan 689-798, Korea \\ a) jysim@unist.ac.kr
}

\begin{abstract}
In this paper, we propose a geometry histogram modification algorithm to increase the visibility of $3 \mathrm{D}$ point models. We define a weak feature as a group of neighboring points yielding the small deviations of normal directions. Geometry histogram is defined as the distribution of the signed distance between a feature point and the locally approximated plane. We equalize and stretch the geometry histogram and move the corresponding feature points accordingly. We also use the OpenGL API for simple and fast rendering of 3D point models. Experimental results show that the proposed algorithm efficiently enhances the geometry contrast of 3D point models by improving the appearance of the weak features.
\end{abstract}

Keywords: geometry contrast enhancement, geometry histogram, histogram modification, 3D point model, graphics hardware

Classification: Electron devices, circuits, and systems

\section{References}

[1] M. Levoy, et al., "The digital Michelangelo project: 3D scanning of large statues," Proc. SIGGRAPH, pp. 131-144, July 2000.

[2] S. Heinzle, G. Guennebaud, M. Botsch, and M. Gross, "A hardware processing unit for point sets," Proc. SIGGRAPH/Eurographics Conf. Graphics Hardware, pp. 21-31, June 2008.

[3] M. Pauly, R. Keiser, and M. Gross, "Multi-scale feature extraction on point-sampled surfaces," Computer Graphics Forum, vol. 22, pp. 281-289, 2003.

[4] A. C. Öztireli, G. Guennebaud, and M. Gross, "Feature preserving point set surfaces based on non-linear kernel regression," Computer Graphics Forum, vol. 28, pp. 493-501, 2009.

[5] M. Pauly, M. Gross, and L. P. Kobbelt, "Efficient simplification of pointsampled surfaces," Proc. IEEE Visualization, pp. 163-170, Nov. 2002.

[6] R. C. Gonzalez and R. E. Woods, Digital image processing, Prentice Hall, 2007.

[7] R. S. Wright, N. Haemel, G. Sellers, and B. Lipchak, OpenGL SuperBible, Addison-Wesley, 2010. 


\section{Introduction}

Point clouds are used to represent highly detailed 3D models [1]. Many techniques have been proposed to efficiently process $3 \mathrm{D}$ point models. While the conventional graphics processing units (GPUs) are used mainly for polygon meshes, a dedicated hardware architecture to 3D point models was also developed [2]. Pauly et al. proposed a robust detection algorithm for line-type features by classifying the points at multiple scales [3]. Öztireli et al. devised a smooth surface representation method for a $3 \mathrm{D}$ point model which preserves sharp geometric features [4]. The conventional processing techniques for 3D point models usually extract the salient geometric features such as edges and corners. However little effort has been made to enhance the weak features, which yield relatively little difference of geometric characteristics between the neighboring points and thus may not be clearly perceived when rendering a $3 \mathrm{D}$ point model.

In this paper, we improve the appearance of the weak features in a 3D point model using geometry histogram modification. We first detect the weak features based on the normal deviation between the neighboring points. For each local region of weak features, a geometry histogram is defined as the distribution of points along the normal direction to the locally approximated plane. In general, a weak feature yields a relatively narrow dynamic range of point positions in the geometry histogram. Therefore, based on the histogram equalization and stretching techniques, we modify the geometry histogram to be more uniformly distributed over a wider dynamic range. The modified 3D point models are rendered through the OpenGL API (Application Program Interface) to exploit GPU for various 3D application systems. Experimental results show that the proposed algorithm effectively changes the weak features to be visible more obviously.

\section{Weak feature extraction}

In 3D point models, while the neighboring points distributed on a flat and smooth surface region have almost the same normal directions, the points on a sharp edge or corner yield the significantly different normal directions from each other. Therefore, we analyze the geometric characteristics of a 3D point model by employing the normal vectors of points. In particular, we observe that the relatively small deviations of normal directions are associated with the points in the weak features, such as shallowly engraved or slightly protruding patterns on a flat surface region. It means that the normal deviation can be used to extract the weak features.

We first assign a normal vector $\overrightarrow{\mathbf{n}}_{i}$ to a point $\mathbf{p}_{i}$ by using the covariance analysis for the K-nearest neighbor points, $\mathbf{p}_{i, j}$ 's $(1 \leq j \leq K)[5]$. Then the normal deviation $\sigma\left(\mathbf{p}_{i}\right)$ is computed at $\mathbf{p}_{i}$ as the average angle between the two normal vectors of $\overrightarrow{\mathbf{n}_{i}}$ and $\overrightarrow{\mathbf{n}_{i, j}}$.

$$
\sigma\left(\mathbf{p}_{i}\right)=\frac{1}{K} \sum_{j=1}^{K} \arccos \left(\frac{\overrightarrow{\mathbf{n}_{i}} \cdot \overrightarrow{\mathbf{n}_{i, j}}}{\left\|\overrightarrow{\mathbf{n}_{i}}\right\|\left\|\overrightarrow{\mathbf{n}_{i, j}}\right\|}\right) .
$$




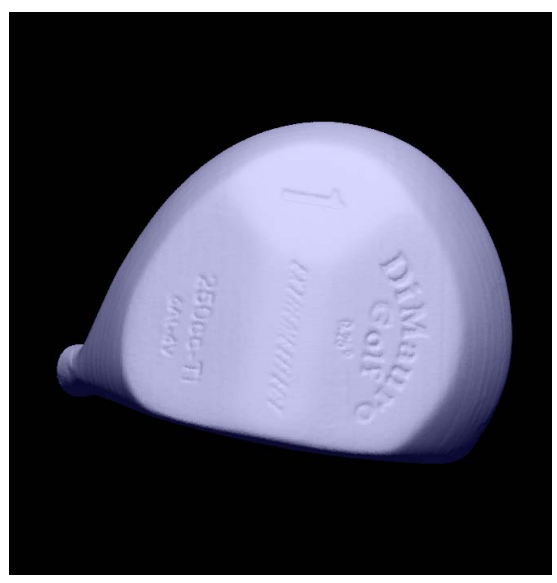

(a)

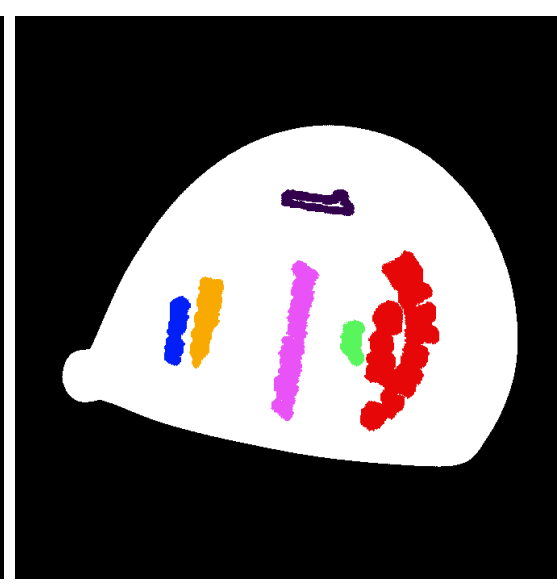

(b)

Fig. 1. Weak feature extraction. The extracted points as weak features are locally grouped into the several regions in the 'Golf-club' model.

Practically, $\mathbf{p}_{i}$ is selected as a point in the weak features when $\sigma\left(\mathbf{p}_{i}\right)$ belongs to a specific range. We employ the 8 -nearest neighbor points to calculate $\sigma\left(\mathbf{p}_{i}\right)$, and adaptively set the user-defined range for the normal deviation according to a $3 \mathrm{D}$ point model.

The selected points as weak features are locally partitioned into several groups using a region growing technique. In addition, we apply the mean filtering for the values of $\sigma\left(\mathbf{p}_{i}\right)$, in order to include the locally adjacent points into a connected region of weak feature. Fig. 1 shows that the several regions are extracted as the weak features from the 'Golf-club' model.

\section{Geometry contrast enhancement}

We introduce a concept of geometry contrast as the differences in the geometric characteristics of a 3D model. The weak features in a 3D point model generally yield degraded geometry contrast, since they are barely distinguishable from the background surface. Histogram equalization and stretching techniques enhance the image contrast by evenly distributing the intensity values [6]. Based on this idea, we increase the visibility of the weak features and enhance the geometry contrast of a 3D point model.

\subsection{Geometry histogram}

For each weak feature, we evaluate the signed distance $d$ from the average position $\overline{\mathbf{p}}$ of the weak feature to a point $\mathbf{p}$ along a specified direction $\overrightarrow{\mathbf{e}}$, which is given by

$$
d=(\mathbf{p}-\overline{\mathbf{p}}) \cdot \overrightarrow{\mathbf{e}} .
$$

Then we define the directional geometry histogram as the probability distribution of $d$ associated with the points in a weak feature. $\overrightarrow{\mathbf{e}}$ is defined as the histogram direction vector and basically changeable. Therefore, for the same group of points, the geometry histogram yields the different distributions according to the selection of histogram direction vector. We set $\overrightarrow{\mathbf{e}}$ as

(c) IEICE 2011

DOI: $10.1587 /$ elex.8.1621 Received August 27, 2011 Accepted September 01, 2011 Published October 10, 2011 
the normal vector of the plane which approximates the distribution of $\mathbf{p}$ 's in a weak feature and passes through $\overline{\mathbf{p}}$, since $\mathbf{p}$ 's yield the smallest variation along this direction.

\subsection{Geometry histogram modification}

Fig. 2 illustrates the geometry contrast enhancement which moves an original point $\mathbf{p}$ to a new position $\widetilde{\mathbf{p}}$ along the corresponding histogram direction $\overrightarrow{\mathbf{e}}$ via

$$
\widetilde{\mathbf{p}}=\mathbf{p}+(\widetilde{d}-d) \overrightarrow{\mathbf{e}},
$$

where $\widetilde{d}=(\widetilde{\mathbf{p}}-\overline{\mathbf{p}}) \cdot \overrightarrow{\mathbf{e}}$. Specifically, the new distance $\widetilde{d}$ is obtained from $d$ by the following transform of histogram modification

$$
\widetilde{d}=\left(\widetilde{d}_{\text {max }}-\widetilde{d}_{\text {min }}\right) C(d)+\widetilde{d}_{\text {min }}
$$

which makes the geometry histogram of $\widetilde{d}$ yield a more uniform distribution than that of $d$.

$C(d)$ represents the cumulative distribution function of $d$, which is used to equalize the geometry histogram. Practically, we compute

$$
C(d)=\frac{\text { number of points with distances less than } d}{\text { total number of points }}
$$

in a selected weak feature.

$\widetilde{d}_{\min }$ and $\widetilde{d}_{\max }$ in (4) denote the lower bound and the upper bound of $\widetilde{d}$, respectively. Note that no specific range of $\widetilde{d}$ is fixed in the geometry histograms, while the range of the intensity values is $[0,255]$ in the histograms of typical images. Therefore, a user can adaptively select a dynamic range of $\left[\widetilde{d}_{\text {min }}, \widetilde{d}_{\text {max }}\right]$ according to the stretching level of geometry histogram. In

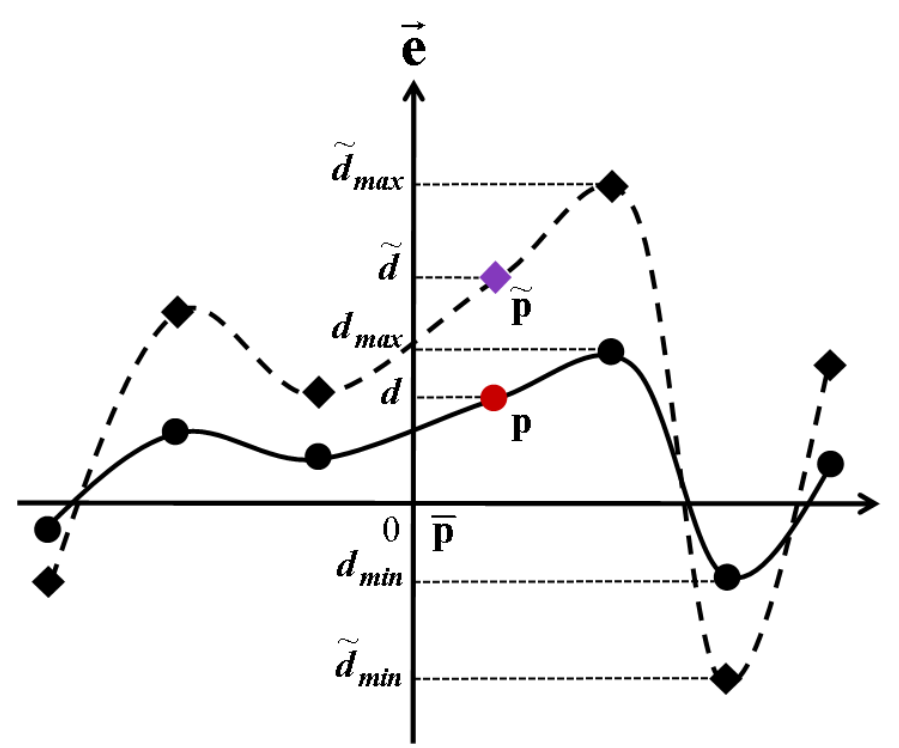

Fig. 2. Geometry contrast enhancement using histogram equalization and stretching along the histogram direction. The solid and dashed curves denote the original and modified geometry, respectively. 
practice, we determine $\widetilde{d}_{\min }$ and $\widetilde{d}_{\max }$ as

$$
\begin{aligned}
& \widetilde{d}_{\min }=\tau d_{\min }, \\
& \widetilde{d}_{\max }=\tau d_{\max },
\end{aligned}
$$

where $\tau$ is the scaling paramter, and $d_{\min }$ and $d_{\max }$ mean the lower bound and the upper bound of $d$ in the original geometry, respectively.

As shown in Fig. 2, the original positions of neighboring points are not clearly distinguishable from each other along the direction $\overrightarrow{\mathbf{e}}$, since the geometry histogram of $d$ yields a compact distribution within a relatively narrow range of $\left[d_{\min }, d_{\max }\right]$ on $\overrightarrow{\mathbf{e}}$. However, $\widetilde{d}$ 's are more unifomly distributed over the wider range of $\left[\widetilde{d}_{\min }, \widetilde{d}_{\max }\right]$, and thus yield a higher geometry contrast along the histogram direction.

\section{Experimental results}

We evaluate the performance of the proposed algorithm on the test 3D point models shown in Fig. 3. Fig. 3 (a) shows the original 'Golf-club' model composed of 209,779 points, and Fig. 3 (b) shows the enhanced model. We observe that the shallowly carved letters and numbers on the surface of the

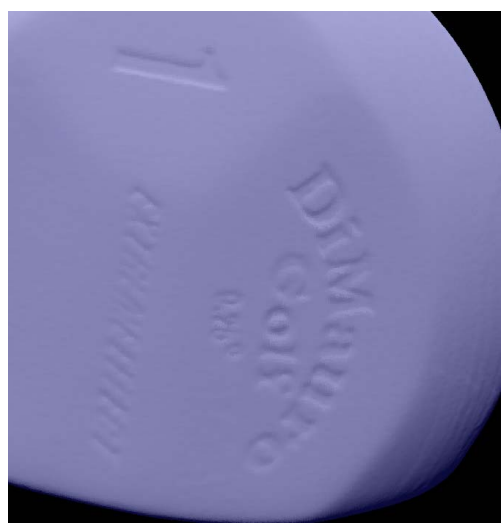

(a)

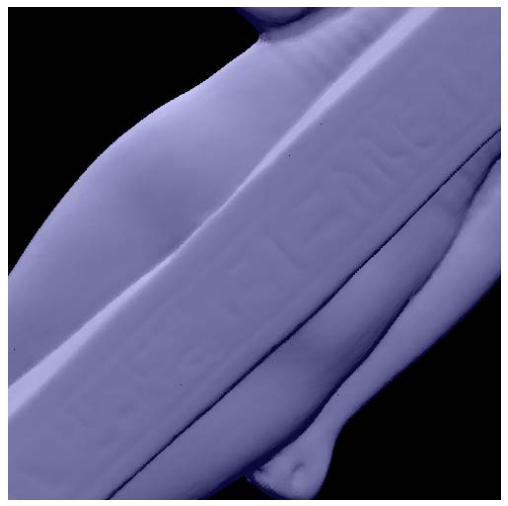

(c)

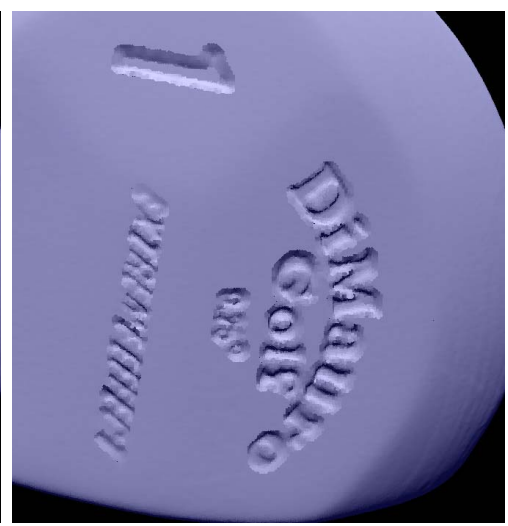

(b)

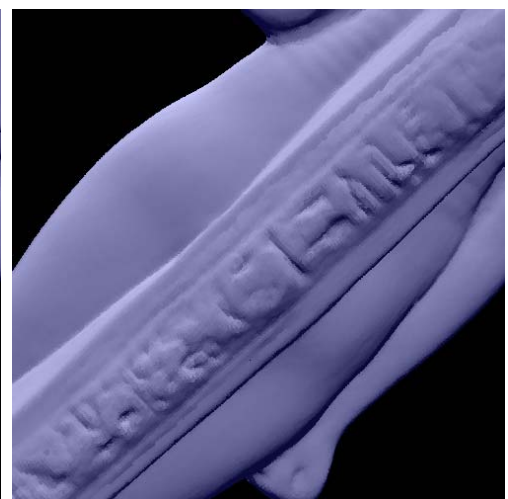

(d)

Fig. 3. Comparative results of geometry contrast enhancement. (a) Original 'Golf-club' model. (b) Enhanced 'Golf-club' model. (c) Original 'Isis' model. (d) Enhanced 'Isis' model. 
original model are clearly appeared in the enhanced model. Fig. 3 (c) shows the original 'Isis' model composed of 187,644 points, where the indistinct patterns are engraved on the back of the model. However, in Fig. 3 (d), we can more easily visualize the patterns by enhancing the geometry contrast. In the experiments, we empirically set the scaling parameter $\tau$ in (6) as 5, which means that the effective range of $\left[\widetilde{d}_{\text {min }}, \widetilde{d}_{\text {max }}\right]$ is 5 times larger than that of $\left[d_{\min }, d_{\max }\right]$.

We also use the glDrawArrays API of OpenGL to facilitate a simple and fast rendering of many points without complex processing [7]. The rendering speed of the 'Golf-club' model and the 'Isis' model is $43 \mathrm{fps}$ and $46 \mathrm{fps}$, respectively, on a system with Intel Core i7 $2.79 \mathrm{GHz}$ CPU, 6 GB RAM, and nVidia Quadro FX 1800 GPU.

\section{Conclusion}

We introduced a novel concept of geometry contrast and proposed a geometry contrast enhancement algorithm for $3 \mathrm{D}$ point models using the histogram modification techniques. The weak features are extracted based on the normal deviation of points. The geometry histogram is defined for each weak feature as the distribution of points along the normal direction to a locally approximated plane. By equalizing and stretching the distribution of points, we increase the visibility of $3 \mathrm{D}$ point models and enhance the geometry contrast.

\section{Acknowledgments}

This research was supported by Basic Science Research Program through the NRF of Korea funded by the Ministry of Education, Science and Technology (2010-0006595). 